\title{
El cluster de la industria del software en Mérida, Yucatán
}

\author{
Manuel J. Caro Encalada \\ Carlos E. Leyva Morales*
}

\begin{abstract}
Resumen
En este trabajo se analizan las empresas de software ubicadas en Mérida, Yucatán, caracterizando su situación, tipo de cluster, potencial de desarrollo y posibles nichos de mercado a futuro. Se encontró que esta industria pertenece al cluster denominado Anclado en el Estado con empresas sólidas en su producción, que poseen una estructura organizacional definida y capital humano de alto nivel de escolaridad y productividad. En materia de comercialización, cuentan con condiciones óptimas al respecto (ventas, distribución, precios y funciones comerciales). El destino principal de sus productos es el país y poco más de la mitad de ellas no tienen dificultades para contratar personal calificado en las tecnologías para atender al mercado interno, aunque sí para el externo. Las finanzas de la mayoría de ellas son sólidas, están equipadas adecuadamente para desarrollar sus procesos productivos y comerciales e internamente presentan más fortalezas que debilidades en sus áreas. Asimismo, son negocios que en su mayoría crecieron en cuanto a su producción, ventas y planta laboral, por lo que esperan que éstos aumenten. Por último, se encontró que existen las condiciones necesarias y suficientes para potenciar el desarrollo de este cluster (entorno regional como nacional); los destinos donde sus productos presentan futuro potencial de ventas son la región sureste y el mercado exterior del país.
\end{abstract}

Palabras clave: Mérida, clusters, tecnologías de información, software.

Fecha de recepción: 06-11-2006

Fecha de aceptación: 05-07-2007

\footnotetext{
* Profesores de la Universidad Autónoma de Yucatán.

Correos electrónicos: mcencala@hotmail.com clmoral@tunku.uady.mx
} 
Manuel J. Caro Encalada y Carlos E. Leyva Morales

\title{
The software industry cluster in Mérida, Yucatán
}

\begin{abstract}
Software enterprises located in Mérida, Yucatán, were analyzed, characterizing their situation, cluster type, development potential and future markets niches. It was found that this industry belongs to state anchorage type, with solid production enterprises, organizational structures defined and high scholarship and productivity levels of human capital. In commercialization they count with optimal levels (sales, distribution, prices and commercial functions). Country is the main destiny of their products and little more half of them have no problems for hiring qualifying personal in technologies for attending inner market not the external one. Most of their finances are solid, adequate equipped for developing their productive and commercial processes and internally have more strengthens than weakness in their areas. They are business that grows mostly about production, sales and labor plant, and wait their increment. Finally, it was found that exists the necessary and enough conditions for potencies development of this cluster (regional and national environment) being destinations were their products presents potential future of sales, the southeast region and international markets.
\end{abstract}

Keywords: Mérida, clusters, information technologies, software.

\section{Introducción}

T a producción de software es una actividad económica que genera un alto valor Lagregado y aporta a la economía productos y servicios esenciales para su modernización. Esta industria se basa en el conocimiento, desarrolla habilidades más allá de la manufactura, propicia la innovación tecnológica, genera empleos bien remunerados, no contamina y requiere de relativamente poco capital para iniciar. La industria del software forma parte del grupo de actividades económicas que conforman las tecnologías de información. Éstas - conjuntamente con la industria de hardware, los servicios y las comunicaciones- constituyen lo que se conoce como Tecnologías de Información y Comunicación (TIC) y su incorporación en los procesos de producción, comercialización, servicios, educación y administración pública es un factor clave para mejorar la competitividad de las organizaciones y los países. ${ }^{1}$

En México existen profundas diferencias en la adopción de tecnologías de información y comunicación. No sólo la penetración y apropiación de las TIC es baja, sino

${ }^{1}$ WITSA (2000), “The Global Information Economy”, en Digital Planet, noviembre. 
que hay una grave desigualdad en la adopción de dichas tecnologías tanto por parte de usuarios como de empresas. Además, existe una baja percepción de los beneficios relacionados a la apropiación de estas tecnologías entre la población; por ejemplo, la penetración de Internet en el mundo es casi dos veces más alta que en México y únicamente uno de cada diez mexicanos tiene acceso a una computadora. Por otro lado, mientras que el $4 \%$ de la población de la región sureste tiene acceso a computadora y el $6 \%$ a Internet; el 23\% de la población de la región centro tienen acceso a computadora y el $21 \%$ a Internet. La desigualdad también se traduce en diferencias en la adopción de la tecnología entre los sectores económicos, el presupuesto de las TIC por empleado se concentra de forma abrumadora en el sector de industria y servicios con un promedio anual de más de 1,300 dólares mientras que en el resto de los sectores éste oscila entre los 350 y los 850 dólares.

Al igual que en la competitividad, la adopción de las Tic es preocupante, no sólo es la distancia entre México y sus competidores, sino que nuestro país ha reaccionado de forma lenta para cerrar dicha brecha; por eso algunos de sus competidores más cercanos (países asiáticos e incluso latinoamericanos) se alejan cada día más rápidamente. Entre los problemas que han hecho que México se rezague se encuentran algunas cuestiones de coyuntura como el tener una de las peores distribuciones de ingreso y un grave rezago educativo; sin embargo, países con peores distribuciones de ingreso y un mayor atraso educativo muestran mayores tasas de penetración de las TIC.

Las principales características que definen la situación actual de la industria de la tecnología de información y comunicación nacional son: número reducido de empresas desarrolladoras de software a la medida, empaquetado y aplicaciones con capacidad para competir internacionalmente, las cuales no han contado con los recursos para atacar los mercados potenciales; el sistema educativo no provee recursos humanos con las características requeridas por la industria; los procesos para el desarrollo de software y el nivel de calidad de las empresas locales deben cumplir con certificaciones y estándares internacionales; el grado de utilización de las TIC en proyectos del sector público no ha servido para fortalecer al sector ni mejorar el nivel de calidad de las empresas locales; no existe un marco regulatorio promotor que facilite la formación de empresas, su desarrollo y acceso a mercados internacionales; las características de la industria no la hacen sujeto de crédito ante la banca comercial o atractiva para la captación de inversiones extranjeras; y, finalmente, que el nivel de competitividad de las empresas de las TIC se mide de acuerdo con las capacidades de los países, además México no se ha posicionado en el mercado mundial. ${ }^{2}$

\footnotetext{
${ }^{2}$ BANCOMEXT y AMITI (2001), "Importancia del desarrollo de tecnologías de información y comunicaciones (TIC) en México", www.claveempresarial.com
} 
Por lo que respecta al estado de Yucatán, la industria de tecnologías de la información (TI) cuenta actualmente con más de diez años de experiencia en el impulso de sus diferentes subsectores, siendo el más avanzado el de desarrollo de programas de software. Se cuenta en total en la entidad con 34 empresas dedicadas al desarrollo de software, con 200 distribuidores de hardware, tres centros de llamadas y un centro de captura de datos. Las principales empresas desarrolladoras de estos productos están ubicadas en la ciudad de Mérida y se encuentran exportando productos y servicios a los Estados Unidos de Norteamérica y a diversos países de Latinoamérica. Se cuenta, también, con siete universidades y 17 institutos tecnológicos, públicos y privados, en los que se desarrollan profesionales para este sector cursando licenciaturas en computación, ingeniería en sistemas, técnicos en programación, entre otros.

En 2002 diferentes instituciones de educación superior y de investigación —en coordinación con el gobierno del estado e industrias locales- crearon el Consejo de la Industria de la Tecnología de la Información (СITI), cuyo objetivo es diseñar e implementar estrategias que impulsen el desarrollo de esta industria en el estado y funcionar como una plataforma para las empresas que deseen ampliar sus horizontes y potenciarlos en el ámbito nacional e internacional. En noviembre de 2003, el gobierno del estado entrega en comodato un edificio a este consejo para dar paso a la creación del ciтi-Centro, facilitando la integración de las empresas de este sector dentro de una misma área común e incluyente para, así, apoyar su desarrollo en la localidad. Este complejo alberga empresas que brindan servicios a grandes consorcios nacionales e internacionales (PEMEX y Honeywell), así como también a pequeñas empresas locales: farmacias, tiendas de artículos fotográficos, restaurantes y dependencias gubernamentales. Otras acciones emprendidas para impulsar el desarrollo de esta industria en el estado han sido: la participación en eventos como т1@Americas $\&$ Prosoft, la Cumbre Internacional de la Industria del Software, la obtención de recursos del Fondo de Apoyo para el Desarrollo de la Industria del Software (Prosoft) y servicios relacionados, así como el aporte de recursos económicos a partes iguales de la federación con el gobierno del estado para fomentar un fondo común destinado a tres proyectos: CITI integración, que unifica como figura jurídica a las empresas que se sumen al proyecto; сіті capacitación, que genera capital humano certificado que consolide contratos con compañías de alto nivel; y СІтІ comercialización, que permitirá consolidar la oferta exportable en тा local a través de Banco de Comercio Exterior, así como fortalecer el mercado doméstico. ${ }^{3}$

Como resultado de todo lo anterior, el estado de Yucatán es actualmente una de las diez entidades invitadas a participar en los fondos del Programa para el Desarrollo de

${ }^{3}$ SEDEINCO (2005), “CiTI-Centro a un año de su inauguración”, en Revista Pulso Económico. 
la Industria del Software, por su contribución al desarrollo de la tecnología de información en México, además de que se visualiza a Yucatán como líder regional y como uno de los tres estados punta del territorio nacional (figura 1).

Figura 1

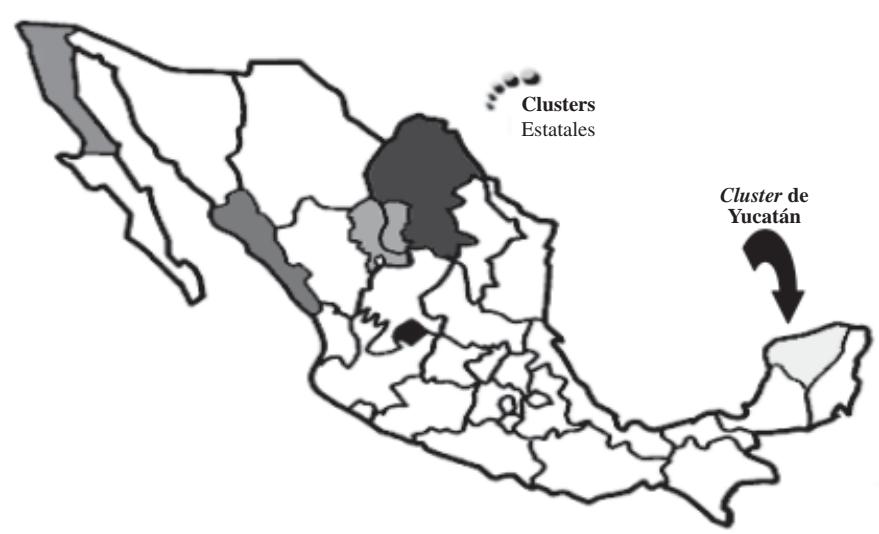

Dado este contexto, se planteó el presente estudio, el cual tuvo como objetivo caracterizar cuál es la situación en que se encuentran actualmente las empresas de software ubicadas en la ciudad de Mérida, Yucatán, que conforman esta industria (tanto en su entorno regional como nacional) y con ello establecer su tipo de cluster, su potencial de desarrollo y la detección futura de posibles nichos de mercado para las mismas.

\section{Metodología de la investigación}

El trabajo se basó en un diseño de comprobación de hipótesis de tipo transversal descriptivo, donde la hipótesis por comprobar fue que la industria del software en Yucatán pertenece al tipo denominado Anclado en el Estado y que existen en la actualidad las condiciones necesarias y suficientes tanto en el entorno regional como nacional para potenciar el desarrollo del cluster de esta industria.

Se empleó como técnica el censo, el cual se basó en las 34 empresas dedicadas a la generación de software identificadas en la zona de estudio y registradas en los listados del cіті y de la Secretaría de Desarrollo Industrial y Comercial. Los tamaños de empresa grande, mediana y pequeña se definieron con base en el número de empleados y el total de ventas de éstas. Las citas para efectuar las entrevistas correspondientes se agendaron en forma directa y mediante llamadas telefónicas; en ellas se encontró que seis empresas se dedicaban a giros diferentes al de interés, por lo que fueron omitidas del universo de estudio. En total se realizaron 18 entrevistas personales y se envió por correo electrónico diez cuestionarios de los cuales sólo de ocho se obtuvo 
respuesta. El levantamiento de la información se efectuó mediante la aplicación de un cuestionario, el cual se conformó con 23 reactivos de opción múltiple y que contó con una prueba previa de aplicación y tiempo de ejecución. Los principales indicadores medidos y analizados para caracterizar esta industria, su tipo de cluster, potencial de desarrollo y nichos de mercado fueron: años de existencia, tamaño de la empresa, capital humano, capital de la empresa, volumen y valor de su producción, volumen y valor de sus ventas, precios de venta, costos de producción (variables y fijos), nivel de rentabilidad, agentes comerciales a quienes vende canales comerciales, funciones comerciales que realiza, estrategias de comercialización, estructura organizativa, evaluación interna, indicadores comparativos e indicadores de confianza.

Finalizado el levantamiento de la información, se procedió al vaciado y procesamiento de la misma en un tabulador diseñado especialmente para el estudio, utilizando para este fin el programa estadístico Excell, con el que se realizaron los cálculos y análisis estadísticos de interés para el estudio.

Los conceptos y principios teóricos considerados en el análisis del trabajo se describen en los siguientes párrafos.

El papel del software para el crecimiento económico. El mundo cada vez más se mueve sobre la base de la tecnología, por ello el desarrollo de productos tecnológicos, entre ellos el software, es una necesidad, pero a su vez una oportunidad de crecimiento económico. De acuerdo con la UNCTAD ${ }^{4}$ (Conferencia de las Naciones Unidas sobre Comercio y Desarrollo), el desarrollo de la industria del software en los países emergentes se puede llevar a cabo a través de dos alternativas de políticas estratégicas. La primera estrategia, denominada de atención de demanda interna, se basa en el uso de software libre como una herramienta para el fortalecimiento de la infraestructura económica con el fin de soportar el desarrollo de otros sectores de la economía, en tanto que la segunda, la generación de un proveedor internacional, se orienta a considerar a la industria del software como sector prioritario por la generación de nuevos empleos y el crecimiento industrial basado en la creación de oferta de software para exportación. Una opción alternativa reciente para el desarrollo de la industria del software en estos países consiste en integrar ambas estrategias bajo un orden secuencial, donde el uso de software libre como herramienta para el fortalecimiento de la infraestructura económica se reemplaza gradualmente por la estrategia de desarrollo de software para exportación.

\footnotetext{
${ }^{4}$ UNCTAD, "Changing Dynamics of Global Computer Software and Services Industry: Implication for Developing Countries”, Naciones Unidas, 2001.
} 
Los clusters. Los nuevos negocios juegan un papel fundamental en la economía, los nuevos negocios están ampliamente involucrados en la actividad innovadora, lo que incrementa la productividad y lleva a incrementar la producción económica de los países. Por otra parte, la aglomeración industrial lleva a la formación de nuevos negocios, y esto puede deberse a que los nuevos negocios pueden abrir en un área con un cluster establecido para operar cerca de un recurso natural clave alrededor del cual el cluster fue inicialmente formado; también puede originarse al establecerse cerca de otros establecimientos similares de tal manera que los miembros de los clusters puedan compartir trabajadores capacitados y maquinaria especializada; y una tercera explicación es que los emprendedores que viven y trabajan en un área con un cluster industrial establecido pueden usar información obtenida localmente para perseguir nuevas oportunidades de negocios. ${ }^{5}$

Michael Porter ${ }^{6}$ menciona que la interacción dentro del círculo competitivo se fortalece y mejora si las organizaciones involucradas están bien localizadas geográficamente, o como se conoce, formando un cluster. Sugiere que las industrias más competitivas de una nación globalmente son también aquellas que se encuentran en un cluster dentro de ella.

Por su parte, Martin y Sunley ${ }^{7}$ indican que el concepto de cluster de Porter es muy genérico en carácter y que es vago e indeterminado para admitir un rango muy amplio de agrupaciones industriales y especializaciones, enlaces entre suministro y deman$\mathrm{da}$, condiciones factoriales, relaciones institucionales, etc., mientras que al mismo tiempo afirman que está basado en lo que argumentan son procesos fundamentales de estrategia de negocios, organización industrial e interacción económica. Por lo tanto, en lugar de ser un modelo o teoría, que puede ser examinada y evaluada, el cluster se ha convertido en un acto de fe y se ha vuelto una manera válida y significativa de pensar sobre la economía nacional y su descomposición en distintas agrupaciones industriales para propósitos de entendimiento y como promoción de la competitividad y la innovación.

En la realidad los clusters varían considerablemente en tipo, origen, estructura, organización, dinámica y trayectoria de desarrollo; por ello, los clusters pueden caracterizarse como redes de producción de firmas fuertemente interdependientes (incluyendo

\footnotetext{
${ }^{5}$ Condo, Arturo (2001), "Desarrollo de cluster competitivos: La competitividad internacional de empresas e industrias", CLACDS.

${ }^{6}$ Porter, Michael E. (2000), "Location, Competition and Economic Development: Local Clusters in a Global Economy", en Economic Development Quarterly 14.

7 Martin R. y P. Sunley (2003), "Deconstructing Clusters: Chaotic Concept or Policy Panacea”, Journal of Economic Geographic, pp. 5-35.
} 
proveedores especializados) enlazadas entre sí en una cadena de producción de valor agregado. En algunos casos, los clusters también incluyen alianzas estratégicas con universidades, institutos de investigación, servicios de negocios de conocimiento intensivo, instituciones de enlace (brokers, consultores) y clientes. ${ }^{8}$

En México son varios los estudios realizados sobre clusters, entre éstos el de Under Kart trata sobre los clusters de automotores y calzado, donde profundiza en las características de la dinámica económica y tecnológica de esos clusters en los estados que revelan especialización de importancia en ellos, abordando los factores sistémicos, incluidos los apoyos de política, de manera que llega a una interpretación de conjunto y a la identificación de los instrumentos de política industrial que han probado efectividad en el desarrollo e integración de estos clusters; el de Mario Cimoli enfatiza que la cooperación es clave si las firmas pretenden capturar los beneficios de las redes y de la innovación; además, señala que todos los procesos de cambio requieren altos niveles tanto de cooperación como de competencia entre las firmas y los otros actores, pero en nuestro país la mayoría de las actividades de producción ha estimulado una demanda por conocimientos y tecnología del extranjero y las compañías han ganado competitividad mediante actividades enfocadas a productos existentes, no a nuevos, lo cual combinado con deficiencias educacionales progresivas ocasiona, en el largo plazo, una depreciación de las competencias del capital humano local e incentivos adversos para desarrollar uniones con centros de investigación locales; por su parte, Alejandro García Macías - quien identifica la existencia de diversos clusters industriales de pequeñas y medianas empresas que necesariamente tienden hacia la especialización productiva de las regiones- intentó demostrar que las relaciones sociales de los empresarios son determinantes para la constitución de estas redes, donde los actores económicos pueden traducir eficientemente su capital social en alternativas de crecimiento para sus empresas.

La información recopilada en el trabajo de campo se analizó con base en los principios teóricos de Markusen sobre el estudio de los clusters, que señalan los tipos de clusters reconocidos en la literatura y sus principales características, los que se presentan en el siguiente cuadro. ${ }^{9}$

8 OECD (1999), "Boosting Innovation: The Cluster Approach".

${ }^{9}$ Markusen, Ann (1996), "Sticky Places in Slippery Space: A Typology of Industrial Districts", en Economic Geography, pp. 293-313. 
Cuadro 1

\begin{tabular}{|c|c|c|c|c|}
\hline & TIPO MARSHALL & $\begin{array}{l}\text { CENTRALIZADO } \\
\text { RADIAL }\end{array}$ & $\begin{array}{l}\text { PLATAFORMA } \\
\text { SATÉLITE }\end{array}$ & ANCLADO EN EL ESTADO \\
\hline $\begin{array}{c}\text { NÚMERO Y } \\
\text { TAMAÑO Y } \\
\text { DISTRIBUCIÓN } \\
\text { DE LAS FIRMAS }\end{array}$ & ATOMISTA & $\begin{array}{c}\text { FIRMAS } \\
\text { CENTRALES } \\
\text { GRANDES Y FIRMAS } \\
\text { ATOMISTAS O } \\
\text { MICRO CLUSTERS } \\
\text { AL MARGEN }\end{array}$ & $\begin{array}{l}\text { UNAS CUANTAS } \\
\text { FIRMAS GRANDES }\end{array}$ & \\
\hline CONECTIVIDAD & $\begin{array}{c}\text { ALTAMENTE } \\
\text { INTERCONECTADO }\end{array}$ & $\begin{array}{l}\text { CONEXIONES DEL } \\
\text { CENTRO A LOS } \\
\text { MÁRGENES }\end{array}$ & $\begin{array}{c}\text { POCAS } \\
\text { INTERCONEXIONES } \\
\text { ENTRE ELLOS Y } \\
\text { CONEXIONES ENTRE } \\
\text { LAS SUCURSALES } \\
\text { Y LAS BASES } \\
\text { CORPORATIVAS } \\
\text { DISTANTES }\end{array}$ & $\begin{array}{c}\text { CONEXIONES ENTRE } \\
\text { LA ORGANIZACIÓN } \\
\text { ESTATAL Y LAS FIRMAS } \\
\text { AL MARGEN CON BASE EN } \\
\text { SUBCONTRATOS }\end{array}$ \\
\hline $\begin{array}{c}\text { ORIENTACIÓN } \\
\text { DEL CLUSTER } \\
\text { CON RESPECTO } \\
\text { A LAS } \\
\text { ECONOMIAS DE } \\
\text { AGLOMERACIÓN }\end{array}$ & $\begin{array}{c}\text { ALTAMENTE } \\
\text { INTERNA: } \\
\text { MERCADO } \\
\text { DE TRABAJO, } \\
\text { ESPECIALIZACION } \\
\text { INCLUYENDO } \\
\text { PROVEEDORES Y } \\
\text { CONOCIMIENTO } \\
\text { COMPARTIDO }\end{array}$ & $\begin{array}{c}\text { ALTAMENTE } \\
\text { EXTERNO, PERO } \\
\text { INTERNALIZADO } \\
\text { (EXCEPTO PARA EL } \\
\text { INTERIOR DE LOS } \\
\text { MICRO-CLUSTERS) }\end{array}$ & $\begin{array}{l}\text { EXTERNO BAJO, } \\
\text { PRINCIPALMENTE } \\
\text { SE BENEFICIA DE } \\
\text { LA LOCALIZACIÓN } \\
\text { EN LA REGIÓN PARA } \\
\text { TOMAR VENTAJA DE } \\
\text { LOS PRIVILEGIOS } \\
\text { OTORGADOS }\end{array}$ & $\begin{array}{l}\text { EXTERNO BAJO, NO } \\
\text { IMPORTANTE A MENOS } \\
\text { QUE SEA EN FORMA DE } \\
\text { MICRO-CLUSTERS }\end{array}$ \\
\hline $\begin{array}{l}\text { MOVILIDAD Y } \\
\text { EMPLEO }\end{array}$ & $\begin{array}{l}\text { ALTA DENTRO DEL } \\
\text { DISTRITO Y BAJA } \\
\text { FUERA DE ÉL }\end{array}$ & $\begin{array}{l}\text { BAJA DENTRO } \\
\text { LAS FIRMAS } \\
\text { CENTRALES. } \\
\text { LEALTAD A } \\
\text { LAS FIRMAS } \\
\text { CENTRALES EN } \\
\text { LUGAR DE AL } \\
\text { DISTRITO }\end{array}$ & BAJA & $\begin{array}{l}\text { TÍPICAMENTE BAJA, } \\
\text { DENTRO DE LAS FIRMAS } \\
\text { DEL ESTADO, PERO CON } \\
\text { IMPORTANTES FLUJOS } \\
\text { DE TRABAJO FUERA DE } \\
\text { LAS UNIVERSIDADES } \\
\text { Y LOS INSTITUTOS DE } \\
\text { INVESTIGACIÓN }\end{array}$ \\
\hline $\begin{array}{c}\text { IMPORTANCIA } \\
\text { DE LA } \\
\text { FORMACIÓN } \\
\text { DE NUEVAS } \\
\text { EMPRESAS }\end{array}$ & $\begin{array}{l}\text { IMPORTANTE PARA } \\
\text { LA DINÁMICA DE } \\
\text { ESPECIALIZACIÓN }\end{array}$ & $\begin{array}{c}\text { BAJA PRESENCIA } \\
\text { DE FIRMAS } \\
\text { CENTRALES } \\
\text { PUEDEN ACTUAR } \\
\text { COMO INCENTIVO } \\
\text { PARA ATRAER } \\
\text { NUEVAS FIRMAS } \\
\end{array}$ & BAJA & $\begin{array}{c}\text { EXTERNALIDADES } \\
\text { POSITIVAS DE } \\
\text { UNIVERSIDADES } \\
\text { E INSTITUTOS DE } \\
\text { INVESTIGACIÓN PUEDEN } \\
\text { SER IMPORTANTES }\end{array}$ \\
\hline $\begin{array}{c}\text { ROBUSTEZ } \\
\text { INSTITUCIONAL }\end{array}$ & $\begin{array}{c}\text { ALTA, } \\
\text { PROVEEDORES } \\
\text { ESPECIALIZADOS } \\
\text { DE EDUCACIÓN Y } \\
\text { ENTRENAMIENTO. } \\
\text { ASOCIACIONES } \\
\text { FUERTES DE } \\
\text { COMERCIO }\end{array}$ & $\begin{array}{c}\text { NO IMPORTANTE } \\
\text { PRESENCIA } \\
\text { DE FIRMAS } \\
\text { CENTRALES } \\
\text { Y DINÁMICAS } \\
\text { IMPULSORAS DEL } \\
\text { CLUSTER }\end{array}$ & $\begin{array}{c}\text { EL ESTADO PUEDE } \\
\text { FINANCIAR } \\
\text { INFRAESTRUCTURAY } \\
\text { SUBSIDIOS }\end{array}$ & NO IMPORTANTE \\
\hline
\end{tabular}




\section{Resultados y análisis de datos}

Del total de empresas que existen en la ciudad de Mérida, Yucatán, relacionadas con la generación de software, el $23 \%$ de ellas se clasifican como grandes, el $15 \%$ como empresas medianas y el $62 \%$ como pequeñas.

Empresas grandes. En materia de producción, éstas son empresas que en promedio tienen 280 empleados, las cuales generan al año un promedio de 580,000 h/hombre en servicios de desarrollo y/o basados en tecnología de información, licencian productos de su propiedad y realizan $10,900 \mathrm{~h} /$ hombre entre consultorios e investigación. De estas empresas, el $66.66 \%$ ha producido los mismos productos desde su apertura hasta la fecha y el $100 \%$ de ellas no ha mantenido el mismo tamaño de planta creciendo en promedio un $375 \%$ desde su creación a la fecha.

En cuanto a su organización, el personal directivo que existe en estas empresas es en promedio de seis personas con un sueldo medio de $\$ 15,500.00$ al mes, el número de personas en mandos medios es de 21 (promedio) devengando sueldos por valor de $\$ 8,000.00$ y el número de empleados es de 259 con sueldos por valor de $\$ 6,000.00$ —no incluye los sueldos más bajos pagados en las empresas de telemercadeo $(\$ 3,000.00)$. Todas las empresas de este tipo cuentan con manual de operaciones.

Con respecto a los indicadores de la situación del capital humano con que cuentan son los siguientes: en todas estas empresas las personas que toman decisiones importantes en las mismas han tomado o recibido capacitación principalmente en materia de habilidades directivas, comercialización y otros aspectos de interés para su desempeño; con relación al nivel de escolaridad, los integrantes de la mesa directiva presentan mayormente estudios de maestría (66.66\%), en tanto que los de los mandos medios en su totalidad tienen estudios de licenciatura. Los empleados, por su parte, presentan más estudios en el nivel medio superior (bachillerato y carrera técnica) aunque también existen algunos empleados con estudios de licenciatura e incluso de maestría.

Por lo que se refiere a la comercialización de sus productos y servicios, la venta de estos bienes se realiza en la empresa principalmente (representando en promedio el $87 \%$ de sus ventas) y sólo en un $66.66 \%$ de estas empresas se realizan ventas vía Internet (representando el 15\% de sus ventas). El momento de la venta es decidido por la solicitud del comprador; los meses en que realizan sus ventas mayores son los de fin de año (noviembre y diciembre) principalmente (66.66\% de los casos), en tanto que los de las ventas menores son los de principio del mismo (enero y febrero). La forma de distribución predominante de los productos es la de entrega directa de 
los mismos; el principal destino de éstos es el país donde la empresa es quien define el precio de venta (sólo el $33.33 \%$ de estas empresas vende al mercado externo). Mayormente, estas empresas venden sus productos directo al comprador sin intermediarios (sólo las empresas que venden al exterior emplean algún tipo de intermediario, mayorista o minorista). En cuanto a las funciones comerciales que realizan, la común a todas ellas resultó ser la presentación y seguimiento de ventas, sólo en el $67 \%$ de éstas ocurrió en forma adicional la promoción y mercadotecnia, y solamente en las empresas que comercializan al exterior fueron donde se encontraron funciones adicionales a las anteriores como la prospección, la identificación y calificación de clientes y la presupuestación por licencia y proyectos.

En lo que concierne a sus indicadores actuales del empleo, ninguna tiene dificultades para contratar personal calificado en las tecnologías requeridas, tanto para atender el mercado interno como el externo; sin embargo, todas ellas consideran necesario organizar cursos de capacitación para sus empleados en materia de aspectos técnicos, administrativos y de redacción. En cuanto a financiamiento, estas empresas manifestaron que no hicieron uso del mismo en el último año.

Con relación a la evaluación interna que se les realizó a estas empresas, ésta mostró los resultados que se presentan en el siguiente cuadro:

Cuadro 2

Recursos humanos. Las fortalezas se encontraron en el reclutamiento, selección, contratación e inducción del personal, de su capacitación y adiestramiento, de los servicios y prestaciones a los empleados, de personal calificado y en la lealtad, experiencia y calidad del personal en general. Las debilidades sólo se identificaron en los sueldos y salarios y en los sistemas de motivación y recompensa.

Finanzas. Las fortalezas identificadas fueron en materia de la rentabilidad (retorno sobre los activos y el capital de la empresa), la calidad de las utilidades ${ }^{10}$ (margen de interés neto, ingresos y eficiencia operativa), la solvencia (capital a activos, capital a pasivos y pasivo a activo) y la capacidad de generación de recursos internos. Las debilidades se dieron en el capital accionario y la estructura de los costos.

Comercialización de sus productos y servicios. Las fortalezas se dieron en materia de introducción y desarrollo de nuevos productos, imagen de marca, conocimiento del mercado, participación en el mercado regional y nacional, la línea de productos, la calidad del producto, la lealtad de los consumidores, los precios de los productos y el servicio y la asistencia técnica. Las debilidades detectadas fueron en la estructura y dinámica de la fuerza de ventas, a la investigación de mercados, a la publicidad y promoción, a las estrategias de mercado y al conocimiento y las habilidades en la gestión de mercadotecnia.

Producción de sus productos y servicios. Las fortalezas se centraron en materia de localización de la planta, su capacidad utilizada, la mano de obra especializada, las condiciones de las instalaciones, equipo y maquinaria, la capacidad instalada, el diseño e ingeniería de productos y la flexibilidad en la producción. Las debilida-

des se identificaron en el control de inventarios y en la disponibilidad de materias primas.

${ }^{10}$ Normalización de la utilidad antes de intereses, impuestos, depreciación y amortización, ésta será más alta si una mayor proporción de la misma se deriva de las transacciones recurrentes regulares. 


\begin{abstract}
Investigación, innovación, desarrollo y tecnología. Las fortalezas se detectaron en innovación en los procesos productivos, innovación en los productos actuales, en la experiencia en la investigación científica, en el uso de tecnología propia, en la introducción de nueva tecnología, en las formas de adquisición de tecnología, en el acceso a fuentes externas de desarrollo tecnológico, en la creatividad y en el desarrollo de nuevos productos. Las debilidades se dieron en la utilización de asesores externos para la investigación, desarrollo y tecnología, las patentes, las habilidades gerenciales en materia de tecnología, innovación e investigación y desarrollo, las instalaciones para la investigación y desarrollo y el financiamiento para el mismo fin.
\end{abstract}

En lo que atañe a los indicadores comparativos entre el primer semestre del año anterior y el primer semestre del actual, todas coincidieron en que su volumen de ventas en el mercado interno aumentó (lo mismo señalaron las empresas que comercializan al exterior); igualmente, aumentó para todas ellas su producción en términos de proyectos, servicios y horas hombre; en cuanto a su planta laboral y su capacidad instalada en la mayoría de estas empresas (67\%), estos indicadores también se incrementaron.

Finalmente, con relación a los indicadores de confianza para los próximos meses, la mayor parte de estas empresas (66.66\%) espera que su nivel de ventas aumente en los próximos seis meses, al igual que su planta laboral y el nivel de inversión en su empresa. En tanto al clima de negocios en la entidad, la mayoría (66.66\%) considera que éste permanecerá invariable y que no les será necesario recurrir a algún financiamiento

Empresas medianas. En materia productiva, éstas son empresas que en promedio cuentan con 35 empleados, las cuales generan al año un promedio de 53, $400 \mathrm{~h} / \mathrm{hom}$ bre en servicios de desarrollo y/o basados en tecnología de información y que licencian productos de su propiedad. El 100\% ha producido los mismos productos desde su apertura hasta la fecha y no han mantenido su tamaño de planta, pues han crecido en promedio un $300 \%$ desde su creación.

En lo referente a su organización, el personal directivo que existe en estas empresas es en promedio de dos personas con un sueldo medio de $\$ 21,500.00$ al mes, el número de personas en mandos medios es de cuatro devengando sueldos por valor de $\$ 13,500.00$ y la cantidad de empleados es de 33 , con sueldos líquidos por valor de $\$ 7,500.00$ cada uno. Como en el estrato anterior, todas estas empresas cuentan con manual de operaciones.

Con relación a los indicadores de la situación del capital humano con que cuentan, en todas estas empresas las personas que toman decisiones importantes en las mismas han adquirido o recibido capacitación mediante cursos de comercialización, administración de proyectos y certificaciones tecnológicas; en cuanto al nivel de escolaridad de los integrantes de estas empresas, los que conforman la mesa directiva presentan principalmente estudios de licenciatura y maestría ( $50 \%$ en cada caso), mientras que los de los mandos medios en su mayoría tienen estudios de licenciatura, aunque tam- 
El cluster de la industria del software en Mérida, Yucatán

bién se detectaron integrantes con estudios de maestría. La mayoría de los empleados, por su parte, posee estudios de licenciatura, aunque también existen empleados con estudios en el nivel medio superior (carrera técnica).

En lo que concierne a la comercialización de sus productos y servicios, el 100\% realiza la venta de éstos principalmente en la empresa (lo que representa el $90 \%$ de sus ventas) y sólo el $50 \%$ lo hace por teléfono (lo que constituye el $10 \%$ de sus ventas). El momento de la venta es decidido en el $60 \%$ de los casos de acuerdo con el programa de ventas del negocio y en un $40 \%$ por la solicitud del comprador. No fue posible en este estrato establecer los meses en que realizan las ventas mayores y/o menores. La forma de distribución de los productos predominante es mediante la entrega directa de los mismos (87.5\%), en la cual el principal destino de éstos es la región y el exterior ( 55 y $45 \%$, respectivamente), donde la empresa es quien mayormente define el precio de venta $(75 \%)$, la otra forma identificada fue la modalidad donde entre empresa y comprador lo definen. Todas estas empresas principalmente venden sus productos directo al comprador sin intermediarios. De las funciones comerciales que realizan, las comunes en todas ellas son: prospección, presentación y seguimiento de ventas e identificación, así como calificación de clientes; en forma adicional, en el $50 \%$ de éstas sólo se presentan la presupuestación por licencia y proyectos y la promoción y mercadotecnia. El 50\% de estas empresas no posee un área dedicada a la plantación estratégica de su comercialización y el otro 50\% sí.

Por lo que respecta a sus indicadores actuales del empleo, todas tienen dificultades para contratar personal calificado en las tecnologías requeridas para atender su demanda externa y también consideran necesario organizar cursos de capacitación para sus empleados en materia de estándares de calidad de la industria que se maneja, cursos de calidad de servicio y certificaciones, cursos de inglés, así como todos los relacionados con actualización tecnológica y modelos para la producción de software.

En cuanto a financiamiento, sólo el 50\% de estas empresas ha recibido algún tipo de apoyo en el último año, proveniente básicamente de la banca de desarrollo, de Bancomext y del gobierno estatal (FIDEI); en las modalidades de crédito de avio y refaccionario manifestaron que no se les presentó problema alguno. La infraestructura que poseen se caracterizó por contar en promedio con instalaciones de $330 \mathrm{~m}^{2}, 47$ equipos de cómputo, 64 bienes muebles, 4 equipos de comunicación y 7 herramientas de software. Sus ingresos por ventas promediaron al año $\$ 6^{\prime} 141,504.00$, con costos anuales de $\$ 4^{\prime} 828,707.00$ (de éstos el $81.6 \%$ lo constituyeron los pagos por servicios a la empresa), lo que les arrojó como resultado utilidades por valor de \$1’312,797.00. 
La evaluación interna realizada mostró como resultados más importantes (cuadro 3):

Cuadro 3

\begin{abstract}
Recursos humanos. Las fortalezas identificadas fueron en el desarrollo profesional del personal, de la lealtad, experiencia y calidad del personal en general; de su capacitación y adiestramiento; de su seguridad e higiene; del reclutamiento, selección, contratación e inducción del personal; de los sueldos y salarios y del personal calificado existente. Las debilidades se encontraron sólo en los servicios y prestaciones de los empleados.
\end{abstract}

Finanzas. Las fortalezas encontradas se dieron en la calidad de las utilidades, la estructura de la administración y organización de las finanzas de la empresa, así como de la actividad financiera y el acceso al financiamiento externo. Las debilidades se encontraron en la calidad de la deuda ${ }^{11}$ (datos financieros al crédito y provisión para pérdida de la deuda), rentabilidad, solvencia, punto de equilibrio, dividendos, capital accionario, capital de trabajo y cuentas por cobrar.

Comercialización de sus productos y servicios. Las fortalezas percibidas se dieron en materia de participación en el mercado regional, la calidad del producto, la imagen de marca, la lealtad de los consumidores, los precios de los productos, las estrategias de mercado y el conocimiento y habilidades en la gestión de mercadotecnia. Las debilidades que se identificaron fueron de estructura y dinámica de la fuerza de ventas, la publicidad y promoción, la participación en el mercado nacional y la introducción y desarrollo de nuevos productos.

Producción de sus productos y servicios. Las fortalezas fueron en materia de capacidad instalada, tecnología del proceso, disponibilidad de materias primas, disponibilidad de piezas y subensamblajes, localización de la planta, condiciones de las instalaciones, equipo y la maquinaria, capacidad utilizada, control de calidad, diseño e ingeniería de productos y habilidades gerenciales en la producción. Las debilidades sólo se encontraron con relación a la automatización de la producción.

Investigación, innovación, desarrollo y tecnología. Las fortalezas detectadas fueron en el uso de tecnología propia, el acceso a fuentes externas de desarrollo tecnológico, las habilidades gerenciales en materia de tecnología, innovación e investigación y desarrollo, la innovación en los productos actuales, las habilidades gerenciales en tecnología, innovación e investigación y desarrollo, la introducción de nueva tecnología, las formas de adquisición de tecnología, la creatividad, la innovación en los procesos productivos y la calidad de los diseños. Las debilidades se centran en el financiamiento para la investigación y desarrollo, en el desarrollo de nuevos productos, en la experiencia en la investigación, en la utilización de asesores externos para investigación científica y en las patentes.

Por otra parte, con base en los indicadores comparativos entre el primer semestre del año actual y el primer semestre del anterior, el 50\% indicó que su volumen de ventas en el mercado interno permaneció igual; en tanto que el otro $50 \%$ mostró que éste disminuyó; en relación con las ventas al mercado externo consideraron que éstas aumentaron y que tuvieron igual comportamiento en su producción en términos de proyectos, servicios y horas hombre; mientras que a su planta laboral y a su capacidad instalada, el 50\% consideró que éstas se incrementaron en el periodo considerado, en tanto que el otro $50 \%$ señaló que permanecieron iguales.

Por ultimo, en relación con los indicadores de confianza para los próximos meses, estas empresas esperan que su nivel de ventas aumente en los próximos seis meses,

${ }^{11}$ Razón que establece la composición de la deuda, en relación con el plazo de cancelación. Cuanto menor sea su valor mejor será para la empresa. 
El cluster de la industria del software en Mérida, Yucatán

al igual que su planta laboral; el 50\% espera que aumente el nivel de inversión en su empresa en tanto que el otro $50 \%$ espera que permanezca igual; en lo que se refiere al clima de negocios en la entidad, estas empresas consideran que éste permanecerá invariable y que les será necesario recurrir a alguna fuente de financiamiento, ya sea del tipo Prosoft-Fondos, Secretaría de Economía para el desarrollo de software o Pyme-Fondos, Secretaría de Economía.

Empresas pequeñas. En cuanto a producción, éstas son empresas que en promedio tienen cinco empleados, las cuales generan al año un promedio de $11,381.5 \mathrm{~h} / \mathrm{hom}-$ bre en servicios de desarrollo y/o basados en tecnología de información y realizan $1,330 \mathrm{~h} / \mathrm{hombre}$ entre consultorios e investigación (el 25\% no realiza este tipo de producción), sólo el $37.5 \%$ de ellas licencian productos de su propiedad. La mayoría de ellas $(62.5 \%)$ no ha producido los mismos productos desde su apertura hasta la fecha; los cambios que han realizado se centran principalmente en materia de nuevos y mejores productos y diferentes estrategias de ventas ( $75 \%$ de estas empresas). También la mayoría de ellas (62.5\%) no han mantenido el mismo tamaño de planta de su negocio han crecido en promedio $124 \%$.

En su organización, del personal directivo que existe en estas empresas el 50\% de los casos está conformado por un directivo con un sueldo medio de $\$ 9,000.00$ al mes, en tanto que el otro $50 \%$ está conformado por dos o tres directivos con un sueldo medio de $\$ 6,250.00$. Sólo el $37.5 \%$ de estas empresas cuenta con personal a nivel de mandos medios, el número de personas en promedio con que cuentan es de dos y devengan sueldos por valor de $\$ 6,000.00$; en tanto el número de empleados que fue de dos a cuatro en la mayoría de ellas (71\%) tuvieron sueldos por valor de $\$ 3,936.00$ en promedio (rango de 2, 550 a 6,000). Sólo el $50 \%$ de las empresas de este tipo cuentan con manual de operaciones y el 50\% que no lo tiene argumentó, como causas de esto, no saber elaborarlo o tenerlo en proceso de desarrollo.

En lo que concierne a los indicadores de la situación del capital humano con que cuentan, en todas estas empresas las personas que toman decisiones importantes en las mismas han tomado o recibido capacitación en materia de habilidades directivas, de comercialización, administrativas, de manejo de equipos, de redes y de tecnología; en cuanto al nivel de escolaridad, los integrantes de la mesa directiva presentan principalmente estudios de licenciatura (62.5\%) y posgrado (sólo el $50 \%$ ), mientras que los de los mandos medios en su mayoría (66.66\%) tienen estudios de licenciatura. De igual manera, la mayoría de los empleados presenta estudios de licenciatura (78.5\%), aunque también se encontró empresas en que existen algunos empleados con estudios en el nivel medio superior e incluso posgrado. 
Por lo que respecta a la comercialización de sus productos y servicios, el 75\% de estos negocios realiza su venta en la empresa principalmente (lo que representa en promedio el $82 \%$ de las ventas que realizan) y sólo el $50 \%$ lo hace además por Internet o mediante visitas a las empresas (lo que constituye aproximadamente el 25\% y $73 \%$ de sus ventas). El momento de la venta es decidido en el $75 \%$ de los casos por la necesidad de dinero de parte de la empresa (lo que significa en promedio el $40.8 \%$ de sus ventas), en un $62.5 \%$ por la solicitud del comprador (lo que equivale el $55 \%$ de sus ventas) y en un $50 \%$ de acuerdo con el programa de ventas (lo cual representa el $65 \%$ de sus ventas). Los meses en que realizan sus ventas superiores, la mayor parte de estas empresas (87.5\%) son en los meses de la primera mitad del año y las ventas menores durante los meses de la segunda mitad (62.5\%). La forma de distribución de los productos predominante es la de entrega directa de los mismos (100\%), el principal destino de éstos es el estado y el país (50 y 62.5\%, respectivamente), donde la empresa es quien mayormente define el precio de venta (75\%); la otra forma identificada fue la modalidad en la que la empresa y el comprador lo definen. La mayoría de estas empresas (87.5\%) vende sus productos directo al comprador sin intermediarios; de las funciones comerciales que realizan, las más comunes a todas ellas resultaron la presentación y seguimiento de ventas, la prospección y la identificación y calificación de clientes (62.5\%), presentándose sólo en el 50\% de éstas en forma adicional la promoción y mercadotecnia; sólo el $62.5 \%$ de estas empresas medianas posee un área dedicada a la plantación estratégica de su comercialización.

Con relación a sus indicadores actuales del empleo, el 50\% de estas empresas tienen dificultades para contratar personal calificado en las tecnologías requeridas (las que venden fuera del estado) y el otro $50 \%$ no. La mayoría de ellas $(75 \%)$ considera necesario el organizar cursos de capacitación para sus empleados en materia de sinergia empresarial, administración de proyectos, cursos de capacidades técnicas, comerciales y administrativas y todos los relacionados con actualización tecnológica (información, cliente servido y multimedia) y modelos para la producción de software.

En cuanto a financiamiento, el $87.5 \%$ de ellas no han recibido algún tipo de apoyo en el último año. Su infraestructura se caracteriza por contar, en el 50\% de ellas, con instalaciones con un tamaño promedio de $36.25 \mathrm{~m}^{2}$, en tanto que el otro $50 \%$ presentó instalaciones que ocupaban $77.5 \mathrm{~m}^{2}$; los otros componentes que conformaron esta infraestructura son: 7 equipos de cómputo, 15 bienes muebles, 6 equipos de comunicación, 11 herramientas de software y un vehículo. Por lo que respecta a sus ingresos por ventas, éstos promediaron al año $\$ 816,595.00$ con costos anuales de $\$ 664,946.00$ (de éstos el 63.6\% lo constituyeron los pagos por servicios a la empresa), lo que dio como resultado utilidades por valor de $\$ 151,649.00$. 
De la evaluación interna los resultados más relevantes se presentan en el siguiente cuadro:

\title{
Cuadro 4
}

\begin{abstract}
Recursos humanos. Las fortalezas se localizaron en el desarrollo profesional, en la capacitación y adiestramiento del personal, en los sueldos y salarios, en los sistemas de motivación y recompensa, en los servicios y prestaciones, en el personal calificado, en la lealtad, en la experiencia y calidad del personal y en la seguridad e higiene. Las debilidades se identificaron principalmente en el reclutamiento, selección, contratación e inducción del personal, a las relaciones sindicales y al personal no calificado.
\end{abstract}

Finanzas. Las fortalezas más importantes fueron la rentabilidad de la empresa, la calidad de las utilidades, la solvencia, la actividad (ventas a total de activos), el punto de equilibrio, los dividendos, el capital de trabajo, la capacidad de generación de recursos internos, la estructura de los costos, las cuentas por cobrar y la estructura de la administración y organización. Las debilidades que se encontraron se centran en el acceso al financiamiento externo y al capital accionario.

Comercialización de sus productos y servicios. Las fortalezas se encontraron en materia de estructura y dinámica de la fuerza de ventas, investigación de mercados, publicidad y promoción, participación en el mercado regional, línea de productos, calidad del producto, introducción y desarrollo de nuevos productos, imagen de marca, lealtad de los consumidores, precios de los productos, conocimiento del mercado, estrategias de mercado, servicio y asistencia técnica y conocimiento y habilidades en la gestión de mercadotecnia. Las debilidades se presentaron en los canales de comercialización y en la participación en el mercado nacional.

Producción de sus productos y servicios. Las fortalezas identificadas se dieron en la localización de la planta, las condiciones de las instalaciones, equipo y maquinaria, la capacidad instalada, la capacidad utilizada, el control de inventarios, el control de calidad, la tecnología del proceso, el diseño e ingeniería de productos, la flexibilidad en la producción, la disponibilidad de materias primas, la disponibilidad de piezas y subensamblajes, la mano de obra especializada, la automatización, la productividad y las habilidades gerenciales en la producción. Es importante señalar que en estas empresas no se encontró debilidad alguna en materia de producción.

Investigación, innovación, desarrollo y tecnología. Las fortalezas se dieron en el uso de tecnología propia, la introducción de nueva tecnología, las formas de adquisición de tecnología, el acceso a fuentes externas para el desarrollo tecnológico, la creatividad, la innovación en los procesos productivos, la innovación en los productos actuales, el desarrollo de nuevos productos, la calidad de los diseños, la experiencia en la investigación científica y en las habilidades gerenciales en materia de tecnología, innovación e investigación y desarrollo. Las debilidades se identificaron en las instalaciones para investigación y desarrollo, el financiamiento para investigación y desarrollo, la utilización de asesores externos para investigación, desarrollo y tecnología y en las patentes.

Por otro lado, en lo que concierne a los indicadores comparativos entre el primer semestre del año actual y el primer semestre del anterior, sólo el 50\% de estos giros coincidieron en que su volumen de ventas en el mercado interno aumentó; el otro $50 \%$ no opinó por ser en su mayoría empresas de reciente creación; para las que venden al exterior el comportamiento de su volumen de ventas aumentó; sólo el 50\% de estas empresas aumentó su producción en términos de proyectos, servicios y horas hombre, al igual que su planta laboral y de su capacidad instalada sólo para el 67\% ésta aumentó, en tanto que para un 33\% ésta permaneció igual.

Finalmente, en lo relativo a los indicadores de confianza para los próximos meses, todas estas empresas esperan que su nivel de ventas aumente en los próximos seis 
meses; sólo el $62.5 \%$ considera que su planta laboral aumentará; el 37.5\% restante espera que ésta permanezca igual; el 75\% cree que aumentará el nivel de inversión en sus empresas, mientras que el $25 \%$ restante opina que permanecerá igual; en lo que se refiere al clima de negocios en la entidad, la mayoría de estas empresas (62.5\%) considera que éste será positivo en su evolución y sólo el 37.5\% señala que permanecerá invariable; y en materia de financiamiento, para el $62.5 \%$ de estas empresas no les será necesario recurrir a alguna fuente de crédito en tanto que el $37.5 \%$ restante sí lo necesitará.

\section{Conclusiones}

Con base en las características anteriormente descritas y los principios teóricos que sustentan la teoría de análisis considerada se puede establecer que:

1. El cluster de la industria de software en la ciudad de Mérida, Yucatán, pertenece al tipo denominado Anclado en el Estado (de acuerdo con la clasificación de Markusen, 1969), puesto que en él están presentes: conexiones entre la organización estatal y las firmas al margen sobre la base de subcontratos; una orientación externa baja del cluster con respecto a las economías de aglomeración y no importante a menos que sea en forma de micro-clusters; una movilidad y empleo típicamente baja dentro de las firmas del estado, pero con importantes flujos de trabajo fuera de las universidades y los institutos de investigación; una importancia de externalidades positivas de universidades e institutos de investigación en la formación de nuevas empresas y una robustez institucional no importante.

2. Las empresas que conforman el cluster de la industria del software en la ciudad de Mérida, Yucatán son sólidas en términos de su producción, pues presentan variedad de productos y servicios, innovación y cambio en los mismos y tasas de crecimiento altas en su tamaño de planta. Asimismo, el cluster está constituido por empresas que cuentan con una estructura organizacional definida y que en su mayoría tiene manual de operaciones. Contando, además, con capital humano de alto nivel de escolaridad (licenciatura) y productividad en todos los rangos de su planta laboral; la capacitación de sus tomadores de decisiones es un elemento central en su programa de desarrollo empresarial.

3. En materia de comercialización, las empresas que conforman el cluster son negocios con condiciones óptimas al respecto porque centran sus ventas en la empresa - donde las modalidades más usuales para decidir el momento de venta se dan en términos de la necesidad de dinero, el programa de ventas y la solicitud del comprador-; distribuyen sus productos a través de la entrega directa, ellos son quienes definen sus precios de venta, venden en forma directa al comprador y todas ellas realizan al menos una fun- 
El cluster de la industria del software en Mérida, Yucatán

ción comercial. Cabe mencionar que el destino principal de sus productos es el país, le sigue en importancia la región y el estado, y el mercado exterior.

4. Poco más de la mitad de las empresas no tienen dificultades para contratar personal calificado en las tecnologías requeridas para la atención del mercado interno, aunque sí para el externo, además, la gran mayoría considera necesario organizar cursos de capacitación para mejorar el desempeño de sus empleados.

5. Las finanzas actuales de la mayoría de las empresas del cluster son sólidas, pues no han requerido de financiamiento alguno en el pasado reciente.

6. En términos de infraestructura, estas empresas están equipadas adecuadamente para desarrollar sus procesos productivos y comerciales e internamente presentan áreas de administración de recursos humanos, de finanzas, de comercialización, de producción y de investigación, innovación y desarrollo tecnológico con más fortalezas que debilidades.

7. Son negocios que en su mayoría crecieron, con relación al año anterior, en términos de su producción, ventas y planta laboral que esperan que éstas en sus empresas aumenten en el próximo año, aunque para poco más de la mitad de ellas el pronóstico con relación al clima de negocios en la entidad es que éste permanecerá invariable.

8. Que en Yucatán la alternativa para el desarrollo de la industria del software ha consistido en integrar las dos estrategias de políticas propuestas por la UNCTAD bajo un orden secuencial, donde el uso de software libre como herramienta para el fortalecimiento de la infraestructura económica estatal se está reemplazando gradualmente por la estrategia de desarrollo de software para exportación.

9. Por lo tanto, se puede afirmar que existen en la actualidad las condiciones necesarias y suficientes tanto en el entorno regional como nacional para potenciar el desarrollo del cluster de esta industria en la ciudad de Mérida, capital del estado de Yucatán, donde los destinos hacia donde sus productos presentan un futuro potencial de ventas son la región y el mercado de exportación.

\section{Bibliografía}

BANCOMEXT у AMITI (2001), "Esquema de apoyo gubernamental a la industria de software", Mimeo.

(2001), "Importancia del desarrollo de tecnologías de información y comunicaciones (TIC) en México", http: www.claveempresarial.com. 
Business Software Alliance (1999), "Forecasting a robust future: An economic study of the us software industry", BSA.

Cimoli, Mario (2001), Desarrollando sistemas de innovación: México en un contexto global, The Continuum International Publishing Group Inc, Londres-Nueva York.

Condo, Arturo (2001), "Desarrollo de cluster competitivos: la competitividad internacional de empresas e industrias", presentación de INCAE (Instituto Centroamericano deAdministración deEmpresas), Caracas, Venezuela, septiembre. http://www.conindustria.org/CLUSTERSCOMPETITIVOSsept2001.pdf.

García Macías, Alejandro (2002), "Redes sociales y "clusters" empresariales", REDES- Revista hispana para el análisis de redes sociales, vol. 1, núm. 6, enero, http://revista-redes.rediris.es. Consulta: mayo, 2007.

Markusen, Ann (1996), "Sticky Places in Slippery Space: A Typology of Industrial Districts", en Economic Geography, Rutgers University, New Brunswick, N.J., pp. 293-313.

Martin R. and Sunley P. (2003), "Deconstructing Clusters: Chaotic Concept or Policy Pancea", en Journal of Economic Geografic, 3, Department of Geography, University of Cambridge, UK, pp. 5-35,

OECD (2004), "Information Technology Outlook", en Directorate for Science, Technology and Industry, París.

OECD (1999), "Boosting Innvation: The Cluster Approach", en Directorate for Science, Technology and Industry, París.

Plan Nacional de Desarrollo 2001-2006, Gobierno de la República Mexicana, México.

Porter, Michael E. (1990), The Competitive Advantage of Nations, The Free Press, Nueva York.

(2002), "Regional Foundations of Competitiveness and Implications for Government Policy", Paper Presented at the Departament de Trade and Industry Workshop on regional Competitiveness, оті, Londres.

(2000), "Location, Competition and Economic Development: Local Clusters in a Global Economy", en Economic Development Quarterly 14, núm. 1 , febrero.

Rabellotti R. Julio (1998). "Recovery of a Mexican Cluster: Devaluation Bonanza or Collective Efficiency", Working paper 71, Institute of Development Studies, Inglaterra. 
El cluster de la industria del software en Mérida, Yucatán

RAmos, Joseph (1999), "Una estrategia de desarrollo a partir de los complejos productivos (clusters) en torno a los recursos naturales. ¿Una estrategia prometedora?", en Revista de la CEPAL, 2, agosto.

SEDEINCO (2005), “CITI-Centro a un año de su inauguración”, en Revista Pulso Económico, mayo, pp. 3-4.

UnCtAD (2002), "Changing Dynamics of Global Computer Software and Services Industry: Implication for Developing Countries", en Technology for Development Series, Naciones Unidas, Nueva York y Geneva, http: www.unctad.org/en/docs/ psitetebd12.en.pdf (Consulta: octubre, 2006).

UnGer, Kurt et al. (2003), "Los clusters industriales en México: especializaciones regionales y la política industrial", documento de trabajo desarrollado para el proyecto CEPAL/GTZ: "Una estrategia de desarrollo de clusters basados en recursos naturales", División de Desarrollo Productivo y Empresarial de la CEPAL, Santiago de Chile, septiembre.

World Information Technology and Services Alliance (witsa), (2000), "Digital planet 2000: The Global Information Economy", resumen ejecutivo, noviembre, http://www.witsa.org/ DP2000SUM.PDF. Consulta: octubre, 2006.

(2003), "Digital planet 2002: The Global Information Economy", en Supply Chain Management portal, North Carolina State University, mayo. 